represents a significant gain in statistical power compared to analysis of individual cohorts. Cohorts in the consortium study a variety of health outcomes, including cancer, respiratory, neurologic, reproductive, allergic disorders, injuries, and overall mortality in association with a wide array of agricultural exposures, including potential health hazards and protective factors of relevance to agricultural populations. AGRICOH, then, offers a rich framework to conduct pooling projects on determinants of health and disease in farmers, farm workers and their families. As of May 2017, 29 cohorts from 5 continents are participating in AGRICOH. The studies, varying in size to address different objectives, are from Australia (2), Canada (2), Chile (1), Costa Rica (1), Denmark (1), France (6), New Zealand (2), Norway (3), Republic of Korea (1), South Africa (2), the UK (1), and the USA (7) (http://agricoh. iarc.fr/). Conceived independently, these cohorts adopted varying definitions of the populations and farm types they study, the approaches to select cohort members from the target populations and the extent of documenting farming related activities and exposures. In the first pooling project in AGRICOH, on application of pesticides and risk of myeloid and lymphoid malignancies, harmonisation of pesticide exposure and cancer data has made it possible to generate combined estimates of cancer-type and individual pesticide associations based on over 300000 farmers and farm workers enrolled in three cohorts from France, Norway and the USA. This project illustrates challenges encountered and solutions adopted to address the study of a ubiquitous exposure suspected of being associated with excess cancer risk in this setting.

\section{$1673 d$ ASSESSING EXPOSURE TO OCCUPATIONAL CHEMICALS IN LARGE-SCALE EPIDEMIOLOGICAL STUDIES ON OCCUPATIONAL CANCERS}

H Kromhout. Environmental Epidemiology Division, Institute for Risk Assessment Sciences, Utrecht University, Utrecht, The Netherlands

\subsection{6/oemed-2018-ICOHabstracts.354}

When pooling data across community-based case-control studies exposure assessment has to be redone in order to overcome the issue of the lowest common denominator. Also when exposure assessment is based on expert judgement, no straightforward approaches exist for calibration and pooling of these exposure estimates. Often though, complete and detailed occupational histories of study participants will be available which can be used for a standardised approach across centres/ studies. For instance in the SYNERGY project we successfully managed to collect actual exposure measurements across Europa and Canada covering almost 4 decades (1970-2010). Based on this wealth of exposure data a JEM could be elaborated with quantitative estimates of level of exposure by job, year and region. Combining this JEM with occupational histories of cases and controls resulted in quantitative exposure histories which allowed derivation of quantitative exposure response relationships for amongst others silica and asbestos. In industry-based cohort studies exposure assessment can often be performed at a more detailed level by ascertaining detailed occupational histories and collecting production characteristics in multiple companies enrolled in a cohort study. By collecting industry-specific measurements with detailed auxiliary information very detailed exposure models can be derived. Consequently these models will allow for quantitative exposure estimates at the detailed level of exposure scenario (rather than at the level of a job). For instance, in the European Asphalt Workers study we were able to estimate quantitatively workers exposure to bitumen fume, organic vapour, and benzo(a)pyrene. Standardisation of exposure assessment tools, approaches and empirical modelling are needed in this day and age where big data will be the norm and will be needed to discern undetected health risks. However, availability of actual measurements of workers' exposure will stay a prerequisite in order to calibrate and validate exposure assessment methods employed in large-scale epidemiological studies on occupational health risks.

\section{3 e POOLED ANALYSIS OF CASE-CONTROL STUDIES ON THE JOINT EFFECTS OF OCCUPATIONAL CARCINOGENS IN THE DEVELOPMENT OF LUNG CANCER}

AC Olsson. International Agency for Research on Cancer, Lyon, France

\subsection{6/oemed-2018-ICOHabstracts.355}

Lung cancer is the most common cancer globally, and tobacco smoking is well established as the main cause. Yet occupational exposures play an important role among exposed workers, especially jointly with smoking. The SYNERGY project was established in 2007 to estimate joint effects of asbestos, respirable crystalline silica, polycyclic aromatic hydrocarbons, chromium/nickel and smoking in the development of lung cancer. Sixteen case-control studies conducted between 1985 and 2010 from Canada, Europe, New Zealand and China were pooled, including 19370 lung cancer cases and 23674 controls with detailed information on tobacco habits and lifetime occupations. Controls were recruited from the general population $(81 \%)$ or hospitals (19\%), and were individually or frequency matched to cases by sex and age. Information was predominantly collected by interviews with the study participants themselves, though next-of-kin respondents were accepted in five of the studies if subjects were unavailable (9.1\% of cases, $6.6 \%$ of controls). The database comprises around 14\% never smokers, whereof 822 cases. Women represent around $20 \%$ or the study population. A quantitative job exposure matrix (SYN-JEM) was created based on exposure measurements from multiple countries together with auxiliary data, covering a time period of more than 50 years. SYNJEM is based on statistical models that predict job-, time-, and region-specific exposure levels. Cumulative exposures (e.g. $\mathrm{ff} / \mathrm{ml}$-years) were calculated for each subject by linking SYNJEM with individual occupational histories. Unconditional logistic regression models were used to estimate odds ratios (OR), 95\% confidence intervals (CI), and trends. The strengths of SYNERGY includes bringing together epidemiologists and exposure assessment experts from around the world to advance occupational cancer epidemiology, 2) power to study small risks, 3) providing quantitative exposure estimates for population-based case-control studies, and 4) allowing subgroup analyses, e.g. by gender, histology and smoking status. 